Review

\title{
Compatibility and incompatibility in hyphal anastomosis of arbuscular mycorrhizal
}

\section{fungi}

Candido Barreto de Novais ${ }^{1}$, Alessandra Pepe ${ }^{1}$, José Oswaldo Siqueira², Manuela Giovannetti ${ }^{1}$, Cristiana Sbrana ${ }^{3 *}$

${ }^{1}$ University of Pisa - Dept. of Agriculture, Food and

Environment, Via del Borghetto, 80 - 56124 - Pisa - Italy.

${ }^{2}$ Federal University of Lavras - Dept. of Soil Sciences,

Câmpus Universitário, C.P. 3037 - 37200-000 - Lavras,

MG - Brazil.

${ }^{3}$ National Research Council/nstitute of Agricultural Biology

and Biotechnology, Via G. Moruzzi, 1 - 56124 - Pisa - Italy.

*Corresponding author <sbrana@ibba.cnr.it>

Edited by: Fernando Dini Andreote
ABSTRACT: Arbuscular mycorrhizal fungi (AMF), which live in symbiosis with $80 \%$ of plants, are not able to grow when separated from their hosts. Spore germination is not host-regulated and germling growth is shortly arrested in the absence of host roots. Germling survival chances may be increased by hyphal fusions (anastomoses), which allow access to nutrients flowing in the extraradical mycelium (ERM). Perfect anastomoses, occurring with high frequency among germlings and the ERM of the same isolate, show protoplasm continuity and disappearance of hyphal walls. A low frequency of perfect fusions has been detected among co-specific genetically different isolates, although fungal nuclei have been consistently detected in all perfect fusions, suggesting active nuclear migration. When plants of different taxa establish symbioses with the same AMF species, anastomoses between ERM spreading from single root systems establish a common mycelium, which is an essential element to plant nutrition and communication. The interaction among mycelia produced by different isolates may also lead to pre-fusion incompatibility which hinders anastomosis formation, or to incompatibility after fusion, which separates the hyphal compartments. Results reported here, obtained by analyses of hyphal compatibility/ incompatibility in AMF, suggest that anastomosis formation and establishment of protoplasm flow, fundamental to the maintenance of mycelial physiological and genetic continuity, may affect the fitness of these ecologically important biotrophic fungi.

Keywords: Glomeromycota, hyphal fusions, incompatible hyphal interactions, protoplasmic flow, nuclear migration
Received June 18, 2016

Accepted July 27, 2016
In $\mathrm{AMF}$, studies on cellular events preceding successful anastomoses opened the way to genetic tests on compatibility/incompatibility, which showed genetic exchange between different genotypes (Croll et al., 2009). Here, we review the different stages of hyphal interactions involved in anastomosis formation, self and non-self recognition and compatible and incompatible responses among hyphae of closely related vs. genetically distant AMF taxa.

Compatible hyphal interactions leading to perfect anastomosis

\section{Asymbiotic mycelium}

In AMF, spores germinate and grow under appropriate physical, chemical and microbiological conditions and give rise to a coenocytic mycelium, 8-70 $\mathrm{mm}$ long, which stops growing within 20 days of germination (see Giovannetti et al., 2010). Anastomosis between hyphae derived from germinated AMF spores (Figure 1A), hereinafter defined as germlings, was first investigated in Funneliformis mosseae (formerly Glomus mosseae) Funneliformis caledonius (formerly Glomus caledonium) and Rhizoglomus intraradices (formerly Glomus intraradices), using time-lapse microscopic studies (Giovannetti et al., 1999). Anastomoses were observed in the same germling, where perfect fusions were formed at rates of 51-57 $\%$ in F. mosseae, $34-54 \%$ in F. caledonius and $58-68 \%$ in 
$R$. intraradices. Such high interconnectedness levels were made possible by the large number of fusions, 0.6-1.3 $\mathrm{cm}^{-1}$ in hyphal length. Additionally, anastomoses widely occurred also between hyphae derived from germlings within the same AMF isolate (34-55 \% and 59-90 \% in $F$. caledonius and $R$. intraradices, respectively) (Giovannetti et al., 1999). In $F$. mosseae fusions ranged from 40 to $85 \%$, depending on isolates (Giovannetti et al., 2003). These results had also been confirmed by studies performed on germlings of Rhizoglomus clarus and Rhizoglomus irregulare (Croll et al., 2009; Cardenas-Flores et al., 2010; De la Providencia et al., 2013). Successive experiments increased communal knowledge of the extensive occurrence of perfect fusions in different species of tropical AMF isolates, showing that the frequency of anastomosis differed in hyphal contacts within the same or different germlings, which were $42 / 73 \%, 33 / 14 \%$, $75 / 64 \%, 80 / 91 \%$ and 9/24 \%, in isolates of Acaulospora scrobiculata, Acaulospora spinosa, Claroideoglomus etunicatum, Glomus formosanum and Rhizoglomus manihotis, respectively (Novais et al., 2013). A recent study revealed different anastomosis behavior amongst AMF genotypes in Glomeraceae, such as $F$. coronatus germlings which had a poorly interconnected mycelium (average anastomosis frequency, 4 \%) (Pepe et al., 2016). Anastomoses were never observed in Gigaspora rosea nor in Racocetra castanea germlings (Giovannetti et al., 1999; Novais et al., 2013) (Figure 2).
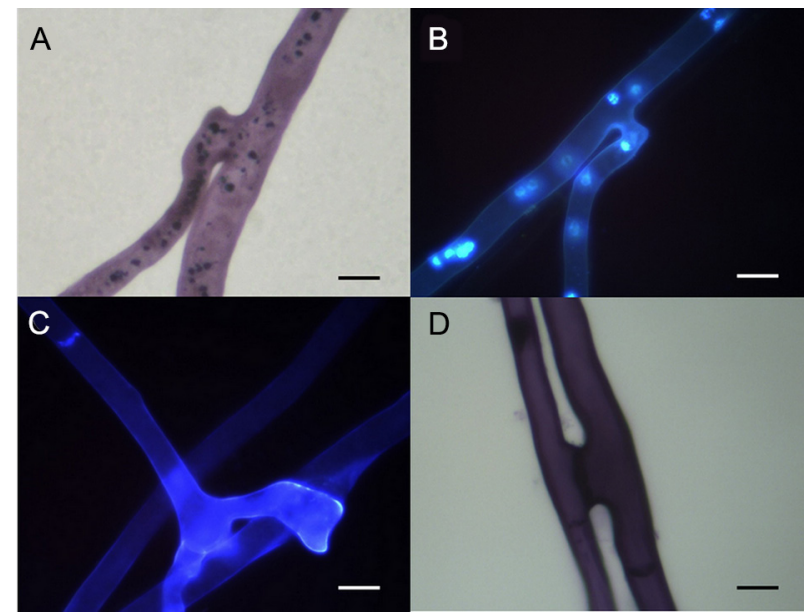

Figure 1 - Light and epifluorescence micrographs of hyphal interactions in arbuscular mycorrhizal fungi. A) perfect anastomosis formed by Funneliformis mosseae hyphae, after staining for Succinate dehydrogenase and with Trypan blue; Scale bar $=5 \mu \mathrm{m}$. B) perfect anastomosis formed by F. mosseae hyphae, showing nuclear mingling after staining with 2,4-diamidinophenylindole; Scale bar $=7 \mu \mathrm{m}$. C) pre-fusion incompatibility in hyphae of Funneliformis coronatus, showing a septum in a tip-swollen hypha after staining with Calcofluor; Scale bar $=6 \mu \mathrm{m}$. D) incompatibility occurring after fusion in F. coronatus, showing septa in empty fused hyphae; Scale bar $=6.5 \mu \mathrm{m}$.
Perfect anastomoses showed a very high degree of cellular compatibility, encompassing the disappearance of hyphal walls, merging of cytoplasm and nuclei and their migration through fusion bridges, leading to protoplasmic continuity between fused hyphae and integration between different mycelia (Giovannetti et al., 1999, 2003; Croll et al., 2009; Novais et al., 2013; Purin and Morton, 2011, 2013). Such cellular events were detected by histochemical localization of succinate dehydrogenase (SDH) activity in hyphal fusions, while time-lapse light microscopy allowed for the monitoring of anastomosis formation, which was achieved in $35 \mathrm{~min}$, and the visualization of protoplasm streaming through fusion bridges, where a mass of cellular particles (possibly nuclei, mitochondria, granules, vacuoles) migrated at the rate of 1.8-2.6 $\mu \mathrm{m} \mathrm{s}^{-1}$. The compatibility between fusing hyphae was supported by evidence of nuclei and associated cytoplasmic microtubules through fusion bridges obtained using 2,4-diamidinophenylindole (DAPI) staining, epifluorescence and immunofluorescence microscopy (Ästrom et al., 1994). Such events of nuclear mingling, originating also from different germlings, indicate that anastomoses allow for the formation of a physiologically and genetically interconnected mycelial network (Figure 1B). Indeed, Croll et al. (2009) demonstrated that anastomoses allowed for genetic exchange between different genotypes and the transfer of genetic markers to the progeny, while Jany and Pawlowska (2010) detected the intermingling of different nuclear lineages within coenocytic hyphae of $C$. etunicatum. Such data were supported by the findings of nuclear and mitochondrial DNA diversity and by recent evidence of mitochondrial gene horizontal transfer and recombination events in Rhizoglomus sp. isolates (Bever and Wang, 2005; Raab et al., 2005; Börstler et al., 2008; Beaudet et al., 2013). Actually it has long been known that in fungi hyphal fusions may give rise to heterokaryons during the parasexual cycle, which have not yet been detected in AMF (Pontecorvo,

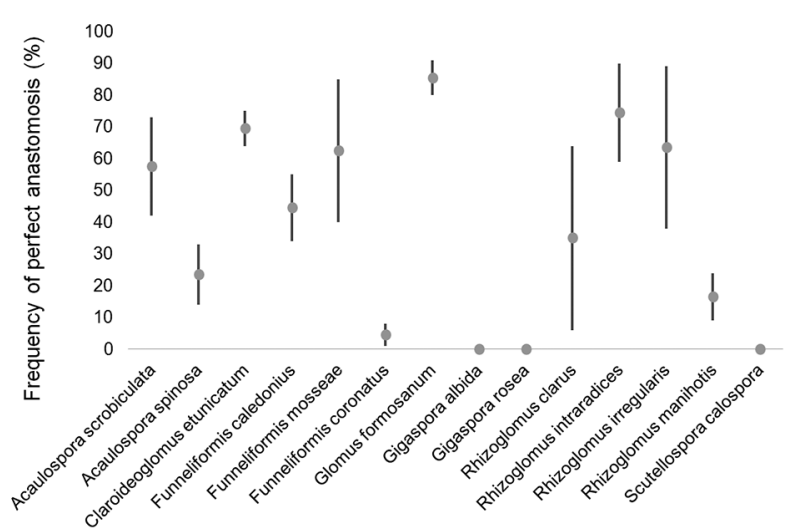

Figure 2 - Graphic summary of bibliographic data reporting frequencies of hyphal contacts resulting in perfect hyphal anastomoses, in spore germlings (asymbiotic stage) of different arbuscular mycorrhizal fungal taxa. 
1956). A large body of molecular studies revealed different patterns of DNA heterogeneity and allowed for the reconstruction of phylogenetic relationships in Glomeromycotan species (Souza et al., 2004, 2005), though the detection of recombination events within single isolates entails multilocus molecular analyses and genomic sequencing in its entirety. Recently, $R$. irregulare transcriptome revealed transcripts putatively involved in meiotic recombination and meiosis-specific proteins (Tisserant et al., 2012), while 51 genes similar to Saccharomyces cerevisiae meiosis genes were described in Glomus spp. (Halary et al., 2011), suggesting that sexual reproduction in AMF could be possible. A putative multi-allelic mating-type locus, containing genes with structural and evolutionary similarity with mating-type loci of other taxa, was found in the genomes of five isolates of $R$. irregulare (Ropars et al., 2016). Additional studies should be carried out in order to investigate whether anastomosis between different AMF genotypes may prelude parasexual cycle or sexual recombination, and thus affect their reproductive success.

A number of authors have detected chemotropism and growth re-orientation before hyphal fusion in $A$. scrobiculata, F. mosseae, F. caledonius, G. formosanum, $C$. etunicatum and $R$. manihotis, (Giovannetti and Sbrana, 2001; Novais et al., 2013), a phenomenon also described in fungi other than AMF, such as Phanerochaete velutina and Stereum spp. (Ainsworth and Rayner, 1989) and Neurospora crassa (Hickey et al., 2002), suggesting that specific recognition signals may be able to modulate anastomosis formation. Nevertheless, information is still lacking on the cytology and biochemistry of the successive developmental steps leading to perfect anastomoses, including cell wall fusion. Further studies should investigate whether specific chemical signals may be active in the control of events preceding contact, such as hyphal fusion competence and positive tropism (Hickey et al., 2002).

\section{Symbiotic mycelium}

Symbiotic mycelium or ERM is the fungal structure wherein mineral nutrients are translocated from soil to plants and sugars transferred from plants to the fungal colony. Thus, a high level of interconnectedness between its hyphae is fundamental to its functional efficiency. Anastomosis occurrence was first visualized in intact ERM formed by $F$. mosseae, using an in vivo model system, which also allowed for the quantification of its growth rate, $738-1067 \mathrm{~mm} \mathrm{~d}^{-1}$ (Giovannetti et al., 2001). In such a system the frequency of fusions was $67-77 \%$, while the number of fusions along hyphae was very high, $0.46-0.51 \mathrm{~mm}^{-1}$. Histochemical localization of SDH activity detected protoplasmic continuity among fusing hyphae, which were $100 \%$ viable, and nuclear migration in anastomosing bridges (Giovannetti et al., 2001). A recent study revealed that AMF genotypes in Glomeraceae differ significantly in perfect anastomosis formation, suggesting fungal symbiont modulation of
ERM interconnectedness, as one isolate of $F$. coronatus consistently showed anastomosis frequencies lower than $8 \%$, when colonising different host plants (Pepe et al., 2016).

The same experimental system provided evidence of anastomosis formation among $F$. mosseae ERM originating from plant roots of different taxa growing nearby. Their frequencies varied according to the different pairings, and ranged from $44 \%$ to $62 \%$ in different combinations between Allium porrum and Solanum melongena or Gossypium hirsutum (Giovannetti et al., 2004). Also in this case perfect anastomoses were confirmed using SDH and DAPI stainings.

An in vitro culture approach, using Ri tRNA-transformed root organ cultures (ROC), detected low anastomosis in the ERM of Glomus hoi, Rhizoglomus proliferus, $R$. irregulare and $R$. intraradices, reaching a maximum of $17 \mathrm{~m}^{-1}$ of hypha in $R$. proliferus (De la Providencia et al., 2005). Gigasporaceae ERM produced rare anastomoses in vitro, confirming previous data obtained in asymbiotic mycelium (Giovannetti et al., 1999). However, $95 \%$ of such intra-hyphal fusions may possibly have represented the result of a healing mechanism (Gerdemann, 1955; De la Providencia et al., 2005).

Further studies are needed in order to understand the relationships between structural ERM traits, such as extent, density and interconnectedness, and the differential fungal foraging ability, functional to efficient nutritional flows in ERM. Indeed, molecular works detected a differential expression of phosphate uptake genes in ERM (Harrison and van Buuren, 1995; Maldonado-Mendoza et al., 2001), showing the structural and functional importance of ERM in phosphate absorption from the soil. A correlation between ERM interconnectedness and plant $\mathrm{P}$ content was found in plants of Medicago sativa colonised by different AMF, confirming the important functional role of highly interconnected ERM in the efficient exploitation of soil resources and differential performances of diverse AMF (Avio et al., 2006). As many other genes encoding proteins for mineral nutrients transport are expressed in ERM, studies focused on ERM functional significance would reveal possible relationships between the expression of mineral transporters in different AMF genotypes and their differential performance.

\section{Asymbiotic vs. symbiotic mycelium}

Recent experimental studies have shown that asymbiotic hyphae are able to fuse with ERM hyphae growing from plants colonized by the same AM fungus, whose frequency was 5-24 \% (Sbrana et al., 2011). This phenomenon may affect survival, viability and the reproductive success of such ancestral obligate biotrophic organisms, which, though lacking a host-regulated spore germination, have survived for 980 million years. Indeed, when germling hyphae do not come into contact with the roots of potential host plants, growth is arrested and protoplasm is withdrawn from the tips to the moth- 
er spores, in order to save precious energy for further germination (Siqueira and Sylvia, 1985). Such evolutionary weakness is compensated for by the capability of germling mycelium to plug into the appropriate ERM and drain plant-derived sugar resources flowing in the mycelial network.

\section{Incompatible hyphal interactions}

Vegetative incompatibility, described in diverse fungal species, occurs when anastomosis formation leads to the intermingling of different nuclear lineages, and involves the death of the relevant common hyphal compartment (Worrall, 1997). Experimental evidence obtained with AMF revealed events of non-self-discrimination leading to incompatibility between AMF hyphae belonging not only to different taxa, but also to co-specific isolates. Such responses occurred either before or after anastomosis.

\section{Pre-fusion incompatibility}

Studies performed on hyphal encounters between AMF germlings of different taxa showed no fusions in combinations among isolates belonging to the genera Funneliformis, Gigaspora and Racocetra (Giovannetti et al., 1999).

When different $F$. mosseae isolates were analysed, most hyphal contacts showed no interference and no anastomoses were formed, while incompatibility preceding fusions, detected in certain interacting hyphae, hindered hyphal fusions (Giovannetti et al., 2003). In such pairings, F. mosseae isolates IN101, BEG25 and AZ225C showed chemotropism, growth re-orientation and branching in the approaching hyphae, while differentiation of lateral tips were observed in the contacted ones. Simultaneously, after growth arrest, the contacting hyphae underwent a series of incompatible responses, such as tip swelling, cell wall thickening, hyphal protoplasm vacuolization and septa formation, separating live from dead hyphal compartments (Giovannetti et al., 2003) (Figure 1C). Nevertheless, the early recognition signals and clues regulating hyphal attraction, tropism and directional growth involved in compatibility/incompatibility remain to be understood.

Pre-fusion incompatibility was detected also in germlings and ERM encounters (Sbrana et al., 2011), indicating that chemical signals leading to incompatible responses are active when hyphae produced at different stages in the AMF life cycle come into contact.

\section{Post-fusion incompatibility}

Incompatible interactions following hyphal fusion were described in Ascomycota, where heterodimers of het (heterokaryon incompatibility) or vic (vegetative incompatibility) proteins (Glass et al., 2004) led to septa formation and protoplasm retraction in anastomosed hyphae.

Post-fusion incompatibility was first detected in AMF during in vitro studies, which revealed that $R$. ir- regulare genetically different clonal lineages could anastomose and exchange specific markers (Croll et al., 2009). The incompatible responses consisting of protoplasm retraction and cross-wall formation in hyphal fusions (Figure 1D), were similar to those described in Ascomycota. Recently, incompatibility following fusion was confirmed in pairings of $R$. irregulare isolates of the Department of Agriculture (Mycology) Ottawa (DAOM) collection (De la Providencia et al., 2013) and during contacts between ERM hyphae and germlings (Sbrana et al., 2011). Differences in gene expression, physiological characteristics and host recognition ability between germling hyphae and ERM, representing highly different developmental phases of AMF, may account for these findings, although determinants of incompatibility are still to be unravelled in AMF. However, such an event does not hinder genetic exchange, since Croll et al. (2009) have shown viable protoplasmic connections allowing for the migration of nuclei even when fusion rates were very low. In such interactions, a peculiar cytological event was observed, namely, the growth of intrahyphal hyphae, also described within damaged hyphae and as double-walled structures in the intraradical mycelium of Glomus fasciculatum (Lim et al., 1983).

An $F$. coronatus isolate, forming low-interconnected mycelium, showed within-isolate incompatible reactions following fusions in 1 to $14 \%$ of hyphal interactions, occurring at both the asymbiotic and symbiotic stage (Pepe et al., 2016), whereas different rates were observed at the same developmental stages in Rhizoglomus clarus (Purin and Morton, 2013). At the symbiotic stage, self-incompatibility may affect AMF fitness and reduce the development of common ERM, with possible impacts on nutrient resources flow and AMF survival chances in soil.

\section{Final Remarks}

Hyphal anastomoses represent key structures for the formation of efficient and interconnected AMF mycorrhizal networks, which are fundamental to AMF survival, to plant/soil nutrient flow and to the maintenance of genetic diversity. Moreover, anastomoses formed between ERM originating from the root system of different host species allows for the formation of wide hyphal networks interconnecting an indefinite number of diverse plants living in the same ecosystem. So far we know almost nothing about the cellular and environmental background allowing two hyphae to anastomose and form an interconnected mycelium. Reported findings suggest a complex hyphal signaling pattern, with common steps and outcomes when hyphal interactions involve similar genotype/fungal life stages (hyphal fusion) or dissimilar genotype/different fungal life stages (hyphal incompatibility). Such variability in AMF anastomosis behaviour suggests the involvement of multiple gene (or multiple allele) variation in determining the degree of compatibility in this group of fungi. 
Further studies should be aimed at improving communal knowledge of AMF hyphal recognition systems and of the impact of different anthropogenic and environmental factors, which may possibly interfere with fungal metabolism, on the formation of highly interconnected and functional ERM.

In particular, the impact of compounds of anthropogenic origin which are commonly found in soil - chemical fertilizers, pesticides and heavy metals - on the anastomosing ability of different AMF should be assessed, in order to detect the best performing AMF strains, characterized by functional traits fundamental to the maintenance of interconnected and effective mycorrhizal networks, to be exploited as suitable biofertilizers and bioenhancers in sustainable agriculture and natural ecosystem management.

\section{Acknowledgments}

Candido Barreto de Novais was supported by a post-doctoral scholarship from CAPES (Coordination for the Improvement of Higher Level Personnel) - Science without Borders (Ciência sem Fronteiras) and Alessandra Pepe was supported by a doctoral scholarship from the University of Pisa.

\section{References}

Ainsworth, A.M.; Rayner, A.D.M. 1989. Hyphal and mycelial responses associated with genetic exchange within and between species of the basidiomycete genus Stereum. Journal of General Microbiology 135: 1643-1659.

Ästrom, H.; Giovannetti, M.; Raudaskoski, M. 1994. Cytoskeletal components in the arbuscular mycorrhizal fungus Glomus mosseae. Molecular Plant-Microbe Interactions 7: 309-312.

Avio, L.; Pellegrino, E.; Bonari, E.; Giovannetti, M. 2006. Functional diversity of arbuscular mycorrhizal fungal isolates in relation to extraradical mycelial networks. New Phytologist 172: 347-357.

Beaudet, D.; Terrat, Y.; Halary, S.; De la Providencia, I.E.; Hijri, M. 2013. Mitochondrial genome rearrangements in Glomus species triggered by homologous recombination between distinct mtDNA haplotypes. Genome Biology and Evolution 5: 1628-1643.

Bever, J.D.; Wang, M. 2005. Arbuscular mycorrhizal fungi: hyphal fusion and multigenomic structure. Nature 433: E3E4.

Börstler, B.; Raab, P.A.; Thiéry, O.; Morton, J.B.; Redecker, D. 2008. Genetic diversity of the arbuscular mycorrhizal fungus Glomus intraradices as determined by mitochondrial large subunit rRNA gene sequences is considerably higher than previously expected. New Phytologist 180: 452-465.

Cardenas-Flores, A.; Draye, X.; Bivort, C.; Cranenbrouck, S.; Declerck, S. 2010. Impact of multispores in vitro subcultivation of Glomus sp. MUCL 43194 (DAOM 197198) on vegetative compatibility and genetic diversity detected by AFLP. Mycorrhiza 20: 415-425.
Carlile, M.J. 1995. The success of the hypha and mycelium. p. 3-19. In: Gow, N.A.R.; Gadd, G.M., eds. The growing fungus. Springer, Amsterdam, The Netherlands.

Croll, D.; Giovannetti, M.; Koch, A.M.; Sbrana, C.; Ehinger, M.; Lammers, P.J.; Sanders, I.R. 2009. Non-self vegetative fusion and genetic exchange in the arbuscular mycorrhizal fungus Glomus intraradices. New Phytologist 181: 924-937.

De la Providencia, I.E.; Nadimi, M.; Beaudet, D.; Morales, G.R.; Hijri, M. 2013. Detection of a transient mitochondrial DNA heteroplasmy in the progeny of crossed genetically divergent isolates of arbuscular mycorrhizal fungi. New Phytologist 200: 211-221.

De la Providencia, I.E.; Souza, F.A.; Fernandez, F.; SéjalonDelmas, N.; Declerck, S. 2005. Arbuscular mycorrhizal fungi exhibit distinct pattern of anastomoses formation and hyphal healing mechanism between different phylogenic groups. New Phytologist 165: 261-271.

Gerdemann, J.W. 1955. Relation of a large soil-borne spore to phycomycetous mycorrhizal infections. Mycologia 47: 619-632.

Giovannetti, M.; Avio, L.; Sbrana, C. 2010. Fungal spore germination and pre-symbiotic mycelial growth: physiological and genetic aspects. p. 3-32. In: Koltai, H.; Kapulnik, Y., eds. Arbuscular mycorrhizas: physiology and function. Springer, Amsterdam, The Netherlands.

Giovannetti, M.; Azzolini, D.; Citernesi, A.S. 1999. Anastomosis and nuclear and protoplasmic exchange in arbuscular mycorrhizal fungi. Applied and Environmental Microbiology 65: 5571-5575.

Giovannetti, M.; Fortuna, P.; Citernesi, A.S.; Morini, S.; Nuti, M.P. 2001. The occurrence of anastomosis formation and nuclear exchange in intact arbuscular mycorrhizal networks. New Phytologist 151: 717-724.

Giovannetti, M.; Sbrana, C. 2001. Self and non-self responses in hyphal tips of arbuscular mycorrhizal fungi. p. 221-231. In: Geitmann, A.; Cresti, M., eds. Cell biology of plant and fungal tip growth. IOS Press, Amsterdam, The Netherlands. (NATO Science Series, I: Life and Behavioural Sciences).

Giovannetti, M.; Sbrana, C.; Avio, L.; Strani, P. 2004. Patterns of belowground plant interconnections established by means of arbuscular mycorrhizal networks. New Phytologist 164: 175-181.

Giovannetti, M.; Sbrana, C.; Strani, P.; Agnolucci, M.; Rinaudo, V.; Avio, L. 2003. Genetic diversity of isolates of Glomus mosseae from different geographic areas detected by vegetative compatibility testing and biochemical and molecular analysis. Applied and Environmental Microbiology 69: 616-624.

Glass, N.L.; Rasmussen, C.; Roca, M.G.; Read, N.D. 2004. Hyphal homing, fusion and mycelia interconnectedness. Trends in Microbiology 12: 135-141.

Halary, S.; Malik, S.B.; Lildhar, L.; Slamovits, C.H.; Hijri, M.; Corradi, N. 2011. Conserved meiotic machinery in Glomus spp., a putatively ancient asexual fungal lineage. Genome Biology and Evolution 13: 950-958.

Harrison, M.J.; van Buuren, M.L. 1995. A phosphate transporter from the mycorrhizal fungus Glomus versiforme. Nature 378: 626-629.

Hickey, P.C.; Jacobson, D.J.; Read, N.D.; Glass, N.L. 2002. Livecell imaging of vegetative hyphal fusion in Neurospora crassa. Fungal Genetics and Biology 37: 109-119. 
Jany, J.L.; Pawlowska, T.E. 2010. Multinucleate spores contribute to evolutionary longevity of asexual Glomeromycota. The American Naturalist 175: 424-435.

Lim, L.L.; Fineran, B.A.; Cole, A.L.J. 1983. Ultrastructure of intrahyphal hyphae of Glomus fasciculatum (thaxter) Gerdemann and Trappe in roots of white clover (Trifolium repens L.). New Phytologist 95: 231-239.

Maldonado-Mendoza, I.E.; Dewbre, G.R.; Harrison, M.J. 2001. A phosphate transporter gene from the extraradical mycelium of an arbuscular mycorrhizal fungus Glomus intraradices is regulated in response to phosphate in the environment. Molecular Plant-Microbe Interactions Journal 14: 1140-1148.

Mikkelsen, B.L.; Rosendahl, S.; Jakobsen, I. 2008. Underground resource allocation between individual networks of mycorrhizal fungi. New Phytologist 180: 890-898.

Novais, C.B.; Sbrana, C.; Saggin, O.J.; Siqueira, J.O.; Giovannetti, M. 2013. Vegetative compatibility and anastomosis formation within and among individual germlings of tropical isolates of arbuscular mycorrhizal fungi (Glomeromycota). Mycorrhiza 23: 325-331.

Pepe, A.; Giovannetti, M.; Sbrana, C. 2016. Different levels of hyphal self-incompatibility modulate interconnectedness of mycorrhizal networks in three arbuscular mycorrhizal fungi within the Glomeraceae. Mycorrhiza 26: 325-332.

Pontecorvo, G. 1956. The parasexual cycle in fungi. Annual Review of Microbiology 10: 393-400.

Purin, S.; Morton, J.B. 2011. In situ analysis of anastomosis in representative genera of arbuscular mycorrhizal fungi. Mycorrhiza 21: 505-514.

Purin, S.; Morton, J.B. 2013. Anastomosis behaviour differs between asymbiotic and symbiotic hyphae of Rhizophagus clarus. Mycologia 12: 589-602.

Raab, P.A.; Brennwald, A.; Redecker, D. 2005. Mitochondrial large ribosomal subunit sequences are homogeneous within isolates of Glomus (arbuscular mycorrhizal fungi, Glomeromycota). Mycological Research 109: 1315-1322.

Ropars, J.; Toro, K.S.; Noel, J.; Charron, A.P.P.; Farinelli, L.; Marton, T.; Krüger, M.; Fuchs, J.; Brachmann, A.; Corradi, N. 2016. Evidence for the sexual origin of heterokaryosis in arbuscular mycorrhizal fungi. Nature Microbiology. 1: 16033.
Sbrana, C.; Fortuna, P.; Giovannetti, M. 2011. Plugging into the network: belowground connections between germlings and extraradical mycelium of arbuscular mycorrhizal fungi. Mycologia 103: 307-316.

Siqueira, J.O.; Sylvia, D.M. 1985. Spores, germination, and germ tubes of vesicular-arbuscular mycorrhizal fungi. Canadian Journal of Microbiology 31: 965-972.

Smith, S.E.; Read, D.J. 2008. Mycorrhizal Symbiosis. 3ed. Academic Press, San Diego, CA, USA.

Souza, F.A.; Declerck, S.; Smit, E.; Kowalchuk, G.A. 2005. Morphological, ontogenetic and molecular characterization of Scutellospora reticulata (Glomeromycota). Mycological Research 109: 697-706.

Souza, F.A.; Kowalchuk, G.A.; Leeflang, P.; van Veen, J.A.; Smit, E. 2004. PCR-denaturing gradient gel electrophoresis profiling of inter- and intraspecies 18S rRNA gene sequence heterogeneity is an accurate and sensitive method to assess species diversity of arbuscular mycorrhizal fungi of the genus Gigaspora. Applied and Environmental Microbiology 70: 1413-1424.

Tisserant, E.; Kohler, A.; Dozolme-Seddas, P.; Balestrini, R.; Benabdellah, K.; Colard, A.; Croll, D.; Silva, C.; Gomez, S.K.; Koul, R.; Ferrol, N.; Fiorilli, V.; Formey, D.; Franken, P.; Helber, N.; Hijri, M.; Lanfranco, L.; Lindquist, E.; Liu, Y.; Malbreil, M.; Morin, E.; Poulain, J.; Shapiro, H.; van Tuinen, D.; Waschke, A.; Azcón-Aguilar, C.; Bécard, G.; Bonfante, P.; Harrison, M.J.; Küster, H.; Lammers, P.; Paszkowski, U.; Requena, N.; Rensing, S.A.; Roux, C.; Sanders, I.R.; Shachar-Hill, Y.; Tuskan, G.; Young, J.P.W.; Gianinazzi-Pearson, V.; Martin. F. 2012. The transcriptome of the arbuscular mycorrhizal fungus Glomus intraradices (DAOM 197198) reveals functional tradeoffs in an obligate symbiont. New Phytologist 193: 755-769.

Worrall, J.J.; Anagnost, S.E.; Zabel, R.A. 1997. Comparison of wood decay among diverse lignicolous fungi. Mycologia 89: 199-219. 